\title{
TBX3 knockdown suppresses the proliferation of hypopharyngeal carcinoma FaDu cells by inducing G1/S cell cycle arrest and apoptosis
}

\author{
YONGJIU HUANG ${ }^{1,2}$, HONGMEI ZHU ${ }^{2}$, XIAOHUI JI ${ }^{3}$, YIN CHEN ${ }^{4}$, \\ YANHUI ZHANG ${ }^{1}$, RUOFEI HUANG ${ }^{1}$, JIN XIE $^{1}$ and PIN DONG ${ }^{1}$
}

\begin{abstract}
${ }^{1}$ Department of Otolaryngology, Head and Neck Surgery, Shanghai General Hospital of Nanjing Medical University, Shanghai 200080; Departments of ${ }^{2}$ Otolaryngology, Head and Neck Surgery, ${ }^{3}$ Pharmacy and ${ }^{4}$ Pathology, Taizhou People's Hospital of Nanjing Medical University, Taizhou, Jiangsu 225300, P.R. China
\end{abstract}

Received January 9, 2019; Accepted August 16, 2019

DOI: $10.3892 /$ ol.2019.11089

\begin{abstract}
The T-box transcription factor family member TBX3 has been demonstrated to participate in the development of various types of cancer, including head and neck squamous cell carcinoma. However, little is currently known about its role in hypopharyngeal carcinoma. In the present study, the involvement of TBX3 in hypopharyngeal carcinoma was investigated. Immunohistochemical assays revealed that TBX3 levels were increased in hypopharyngeal carcinoma compared with normal tissue samples, accompanied by upregulated $\mathrm{N}$-cadherin and downregulated E-cadherin. Lentivirus-mediated TBX3 knockdown efficiently suppressed its expression and inhibited the proliferation of FaDu cells. The opposite was observed in TBX3-overexpressing FaDu cells. These results indicate that TBX3 is essential for FaDu cell proliferation. Furthermore, TBX3 silencing led to a disturbance of the cell cycle, leading to a decrease in the $G_{1}$ phase and an increase in the $S$ phase. In addition, apoptosis was enhanced following TBX3 knockdown. The present results suggest TBX3 as a potential therapeutic target in hypopharyngeal carcinoma.
\end{abstract}

Correspondence to: Dr Pin Dong, Department of Otolaryngology, Head and Neck Surgery, Shanghai General Hospital of Nanjing Medical University, 650 Songjiang Road, Shanghai 200080, P.R. China

E-mail: dongpin64@aliyun.com

Abbreviations: TBX3, T-box transcription factor TBX3; shRNA, short hairpin RNA; RT-qPCR, reverse transcription-quantitative PCR; PI, propidium iodide; p57KIP2, cyclin-dependent kinase inhibitor 1C; PTEN, phosphate and tensin homolog; CDK, cyclin-dependent kinase

Keywords: T-box transcription factor TBX3, proliferation, E-cadherin, $\mathrm{N}$-cadherin, hypopharyngeal carcinoma

\section{Introduction}

Hypopharyngeal carcinoma, which originates in the mucosal epithelia of the hypopharynx, accounts for $5 \%$ of head and neck cancer cases worldwide (1-3). Once diagnosed, this disease has limited treatment options and a poor prognosis (4). Despite the combination of surgery, radiotherapy and chemotherapy benefiting the patients, the overall 5-year survival rate remains $<20 \%$ (5-7). Therefore, there is a constant need to develop novel and effective therapeutic targets for hypopharyngeal carcinoma.

The T-box transcription factor family, which comprises TBX1, TBX2 and TBX3, serves an important role in embryonic development. TBX3 is widely expressed in various tissues and is associated with the pluripotency of embryonic stem cells (8-10). Overexpression of this protein has been demonstrated to be associated with various types of cancer, including breast cancer (11), gastric cancer (12), colorectal cancer (13), bladder cancer (14), head and neck cancer (15) and melanoma (16). Ectopic TBX3 expression promotes the growth and invasion of gastric cancer (12). Mechanistically, TBX3 accelerates papillary thyroid carcinoma cell proliferation by potentiating polycomb repressive complex 2-mediated cyclin-dependent kinase (CDK) inhibitor 1C (p57KIP2) repression. It also drives the growth of sarcoma by suppressing CDK inhibitor 1 (p21) (17). In addition, TBX3 is targeted by microRNA (miR)-17-92 and miR-206, contributing to their suppressive role in pancreatic and breast cancer stem cell viability $(18,19)$. These findings suggest that targeting TBX3 may be helpful in treating patients with cancer. However, the role of this factor in hypopharyngeal carcinoma remains largely unclear.

In the present study, TBX3 was identified as a potential oncogene in hypopharyngeal carcinoma. Its upregulation was observed in hypopharyngeal carcinoma samples when compared with normal tissue samples. The silencing of TBX3 caused cell cycle arrest at the $\mathrm{S}$ phase and increased apoptosis, potentially contributing to the suppressed proliferation of TBX3-knockdown hypopharyngeal carcinoma FaDu cells. By contrast, ectopic TBX3 expression led to an increased 
viability of FaDu cells. Therefore, this transcription factor maybe a promising target for the treatment and monitoring of hypopharyngeal carcinoma.

\section{Materials and methods}

Patient information. Samples from 30 patients (25 male and 5 female) with hypopharyngeal carcinoma and the adjacent tissues were collected from the Taizhou People's Hospital (Taizhou, China) between January 2010 and June 2015. The adjacent non-cancerous tissues were obtained $2 \mathrm{~cm}$ away from the cancer sites. The median age of the patients at the time of surgery was 64.63 years (range, $41-76$ years). Written informed consent was obtained from all patients and the study was approved by the Ethics Committee of the Taizhou People's Hospital.

Immunohistochemical analysis of clinical hypopharyngeal cancer and normal tissues. Human hypopharyngeal cancer and normal hypopharyngeal tissue samples were fixed with $4 \%$ formalin for $24 \mathrm{~h}$ at room temperature and embedded in paraffin ( $5 \mu \mathrm{m}$ thick). The tissues were then subjected to immunohistochemical analysis for TBX3, E-cadherin and N-cadherin. Briefly, the sides were deparaffinized in xylene and hydrated in a graded alcohol series (100, 85 and 75\%). Antigens were retrieved using citrate buffer at $95^{\circ} \mathrm{C}(\mathrm{pH} 6)$, and $3 \%$ hydrogen peroxide was used for endogenous peroxidase blocking, followed by incubation with $10 \%$ goat serum (Abcam) at room temperature for $1 \mathrm{~h}$. The slides were then incubated with the primary antibody at $4^{\circ} \mathrm{C}$ overnight. The incubation with the secondary antibodies was performed at room temperature for $30 \mathrm{~min}$. After staining with 3,3'-diaminobenzidineat room temperature for $20 \mathrm{~min}$, sections were counterstained with hematoxylin at room temperature for $5 \mathrm{~min}$. Images of protein expression were captured using a Zeiss microscope using the brightfield lens at x100 and x400 magnification. Immunostaining scores were analyzed using Image-Pro Plus version 4.1 software (Media Cybernetics, Inc.). The extent of protein expression was graded as follows: Negative, 0 ; weak, 1; moderate, 2; and strong, 4 . The extent of staining was grouped according to the percentage of cells with high staining in the cancer nest: Negative, $0 ; 1-25 \%, 1 ; 26-50 \%$, $2 ; 51-75 \%, 3$; and $76-100 \%$, 4 . The final score of staining was the sum of the protein expression grade and the extent of staining score. The primary antibodies for TBX3 (rabbit polyclonal; 1:200; cat. no. ab99302) and N-cadherin (rabbit polyclonal; 1:600; cat. no. ab76057) were purchased from Abcam, and the primary antibody for E-cadherin (mouse monoclonal; 1:100; cat. no. 14472) was purchased from Cell Signaling Technology, Inc. The horseradish peroxidase-conjugated secondary antibodies (goat anti-rabbit IgG; cat. no. sc-2004; 1:100; and goat anti-mouse IgG; cat. no. sc-2005; 1:100) were from Santa Cruz Biotechnology, Inc.

Cell culture. FaDu and 293T cells (American Tissue Culture Collection) were cultured in DMEM (Hyclone; GE Healthcare Life Sciences) supplemented with $10 \%$ fetal bovine serum (Gibco; Thermo Fisher Scientific, Inc.) and 1\% penicillin and streptomycin solution (Corning Life Sciences) at $37^{\circ} \mathrm{C}$ with $5 \% \mathrm{CO}_{2}$. Cells were passaged when they had reached $80 \%$ confluence.
TBX3 knockdown in hypopharyngeal cancer cells. pGCSIL-GFP lentivirus vectors were used for TBX3 knockdown, and pHelper1.0 and Helper2.0 served as the packaging vectors. All the vectors and short hairpin RNA (shRNA) targeting TBX3 (shTBX3) and non-targeting shRNA (shCtrl) lentivirus particles were designed and purchased from Shanghai GeneChem Co., Ltd. The sequences were as follows: shTBX3, 5'-TGCTGATGACTGTCGTTAT-3'; and shCtrl, 5'-TTCTCCGAACGTGTCACGT-3'. Lipofectamine ${ }^{\circledR} 2000$ (Invitrogen; Thermo Fisher Scientific, Inc.) was used to transfect the vectors $(1 \mu \mathrm{g} / \mathrm{ml})$ into $293 \mathrm{~T}$ cells. Virus supernatants were collected and used for the infection of $\mathrm{FaDu}$ cells. After $72 \mathrm{~h}$ of infection, the knockdown efficiency was determined using reverse transcription-quantitative PCR (RT-qPCR) and western blotting.

TBX3 overexpression in hypopharyngeal cancer cells. The open reading frame sequence of the TBX3 gene (accession number: NM_016569) was inserted into the pCDH lentivirus vectors (purchased from Shanghai GeneChem Co., Ltd.) between the BamHI and SacI sites. 293T cells were used for virus packaging, using Lipofectamine ${ }^{\circledR} 2000$. The virus supernatants with pCDH-TBX3 (OE-TBX3) or pCDH-empty (OE-Empty) were collected and used for infection of $\mathrm{FaDu}$ cells, three times (three days per time). After $72 \mathrm{~h}$ of infection with the lentivirus, the efficiency of transfection was determined by RT-qPCR and western blotting.

Western blot analysis. Total protein was extracted from the cancer cells using RIPA buffer (Beyotime Institute of Biotechnology), and the concentration was measured using a Pierce ${ }^{\mathrm{TM}}$ BCA protein assay kit (Thermo Fisher Scientific, Inc.). The protein samples ( $40 \mu \mathrm{g}$ per lane) were separated via SDS-PAGE (12\% gel) and transferred onto PVDF membranes (Merck KGaA). The membranes were blocked with 5\% skimmed milk at room temperature for $1 \mathrm{~h}$ and then incubated with primary antibodies overnight at $4^{\circ} \mathrm{C}$. Subsequently, the membranes were incubated with secondary antibodies at room temperature for $3 \mathrm{~h}$. The primary antibodies against TBX3 (rabbit polyclonal; cat. no. ab99302),p21 (rabbit monoclonal; cat. no. ab109520), p57KIP2 (rabbit monoclonal; cat. no. ab75974), CDK inhibitor 1B (p27; rabbit monoclonal; cat. no. ab32034) and N-cadherin (rabbit polyclonal; cat. no. ab76057) were purchased from Abcam (all 1:1,000). The primary antibodies against E-cadherin (mouse monoclonal; cat. no. 14472), CDK2 (rabbit monoclonal; cat. no. 2546), CDK4 (rabbit monoclonal; cat. no. 12790), proliferating cell nuclear antigen (PCNA; rabbit monoclonal; cat. no. 13110), cyclin E (rabbit monoclonal; cat. no. 20808), Bcl-2 (mouse monoclonal; cat. no. 15071), caspase 3 (rabbit polyclonal; cat. no. 9662), poly (ADP-ribose) polymerase (PARP; rabbit monoclonal; cat. no. 9532) and GAPDH (rabbit monoclonal; cat. no. 5174) were purchased from Cell Signaling Technology, Inc. (all 1:1,000). Following incubation with the secondary antibodies, the proteins were detected by chemiluminescence (Thermo Fisher Scientific, Inc.). The secondary antibodies (goat anti-mouse IgG; cat. no. sc-2005; and goat anti-rabbit IgG; cat. no. sc-2004) were obtained from Santa Cruz Biotechnology, Inc. (both 1:5,000). Signals were visualized using Pierce ${ }^{\mathrm{TM}}$ enhanced chemiluminescence western blotting substrate (Thermo Fisher Scientific, Inc.), and 
developed using Blue Devil Auto-radiography film (Tanon Science and Technology Co., Ltd.).

Total RNA isolation and RT-qPCR. Total RNA was extracted from FaDu cells transfected with shCtrl or shTBX3, using TRIzol ${ }^{\circledR}$ reagent (Invitrogen; Thermo Fisher Scientific, Inc.). A total of $1 \mu \mathrm{g}$ RNA was subjected to RT using the One Step RT-PCR kit (Takara Biotechnology Co., Ltd.), according to the manufacturer's protocol. RT-qPCR was performed to determine the relative amounts of the transcripts using SYBR master mix (Beijing Transgen Biotech Co., Ltd.) on an IQ5 Real Time PCR System (Bio-Rad Laboratories, Inc.). The thermocycling conditions were as follows: Initial denaturation, $95^{\circ} \mathrm{C}$ for $3 \mathrm{~min}$; followed by 40 cycles of denaturation at $95^{\circ} \mathrm{C}$ for $15 \mathrm{sec}$, annealing at $60^{\circ} \mathrm{C}$ for $30 \mathrm{sec}$ and elongation at $72^{\circ} \mathrm{C}$ for $30 \mathrm{sec}$. GAPDH served as the internal normalization control. The primer sequences are as follows: TBX3 forward, 5'-TGAACTCAACAGCCGCTCCTC-3' and reverse, 5'-CTT CCAAGCCGCTAACCAACC-3'; and GAPDH forward, 5'-TGACTTCAACAGCGACACCCA-3' and reverse, 5'-CAC CCTGTTGCTGTAGCCAAA-3'. The $2^{-\Delta \Delta C q}$ method was used for quantification of the relative mRNA expression levels (20).

MTT assay. shCtrl- and shTBX3-transfected FaDu cells were seeded in 96-well plates at a density of $3 \times 10^{3}$ cells/well and cultured and viability was measured every $24 \mathrm{~h}$ for 5 days. The cells were washed with PBS and incubated with $0.5 \mathrm{mg} / \mathrm{ml}$ MTT solution at $37^{\circ} \mathrm{C}$ for $4 \mathrm{~h}$. MTT reagent was removed and DMSO was added to each well. Subsequently, the cell viability was determined by the measurement of the optical density at $570 \mathrm{~nm}$.

Cell cycle assay. Propidium iodide (PI; Sigma-Aldrich; Merck $\mathrm{KGaA}$ ) staining was employed to determine cell cycle progression. Hypopharyngeal cancer FaDu cells expressing shCtrl or shTBX3 lentivirus were seeded at a density of $5 \times 10^{5}$ cells/well in a 6-well culture plates. The cells were harvested, washed twice with ice-cold PBS, fixed with $70 \%$ ice-cold ethanol overnight and rehydrated in PBS at $4^{\circ} \mathrm{C}$ for $30 \mathrm{~min}$. Subsequently, PI staining was performed at $37^{\circ} \mathrm{C}$ for $30 \mathrm{~min}$ and fluorescence activated cell sorting on a flow cytometer (FACSCalibur; Becton, Dickinson and Company) was used to determine the PI absorbance of the indicated cells. The cell cycle phases were analyzed using ModFit software (version 2.0; Becton, Dickinson and Company).

Apoptosis assay. The Annexin V-APC apoptosis detection kit (eBioscience; Thermo Fisher Scientific, Inc.) was used to detect the apoptosis of shCtrl- and shTBX3-transfected FaDu cells, according to the manufacturer's protocol. In brief, $\mathrm{FaDu}$ cells transfected with shCtrl or shTBX3 were washed with PBS three times and re-suspended using $100 \mu \mathrm{l}$ staining buffer. The cells were then incubated with $5 \mu$ l Annexin V-APC for 15 min. Flow cytometry (FACSCalibur; Becton, Dickinson and Company) was used to detect apoptosis. FlowJo Vx 10.0 software was used to analyze the results (FlowJo LLC).

Statistical analysis. All the data in the present study were analyzed with SPSS software (version 22.0; IBM Corp.). All statistical data are presented as the mean \pm standard devia-
Table I. Association between the expression of TBX3 and clinicopathological factors in patients with hypopharyngeal carcinoma.

\begin{tabular}{|c|c|c|c|c|}
\hline \multirow[b]{2}{*}{ Clinical factors } & \multirow[b]{2}{*}{ No. of patients } & \multicolumn{2}{|c|}{$\begin{array}{c}\text { TBX3 } \\
\text { expression }\end{array}$} & \multirow[b]{2}{*}{ P-value } \\
\hline & & High & Low & \\
\hline Age & & & & $>0.999$ \\
\hline$<65$ years & 16 & 12 & 4 & \\
\hline$\geq 65$ years & 14 & 10 & 4 & \\
\hline Sex & & & & $>0.999$ \\
\hline Male & 25 & 18 & 7 & \\
\hline Female & 5 & 4 & 1 & \\
\hline Differentiation & & & & 0.210 \\
\hline Low & 10 & 9 & 1 & \\
\hline Moderate/High & 20 & 13 & 7 & \\
\hline T stage & & & & $>0.999$ \\
\hline $1-2$ & 18 & 13 & 5 & \\
\hline $3-4$ & 12 & 9 & 3 & \\
\hline $\begin{array}{l}\text { Lymphatic } \\
\text { metastasis }\end{array}$ & & & & 0.391 \\
\hline No & 8 & 7 & 1 & \\
\hline Yes & 22 & 15 & 7 & \\
\hline
\end{tabular}

TBX3, T-box transcription factor TBX3; T, tumor.

Table II. Immunohistochemical staining score of TBX3, E-cadherin and N-cadherin.

\begin{tabular}{lcccc}
\hline Variable & $\mathrm{N}$ & TBX3 & E-cadherin & N-cadherin \\
\hline Normal & 30 & $5(0-7)$ & $7(5-7)$ & $0(0-2)$ \\
Cancer & 30 & $7(5-7)$ & $5(3-7)$ & $3(0-6)$ \\
P-value & & $<0.001$ & $<0.001$ & $<0.001$ \\
\hline
\end{tabular}

TBX3, T-box transcription factor TBX3.

Table III. Correlation between the expression of TBX3, E-cadherin and N-cadherin.

\begin{tabular}{lrr}
\hline Variable & TBX3 & E-Cadherin \\
\hline TBX3 & & \\
E-cadherin & $-0.389^{\mathrm{a}}$ & \\
N-cadherin & 0.105 & -0.010 \\
\hline
\end{tabular}

${ }^{\mathrm{a}} \mathrm{P}<0.05$. Negative correlation between $\mathrm{TBX} 3$ and E-cadherin $\left(\mathrm{r}^{2},-0.389 ; \mathrm{P}=0.034\right)$. TBX3, T-box transcription factor TBX3.

tion of $\geq 3$ independent experiments. Comparisons between two groups were performed using Student's t-test or Fisher's 
A

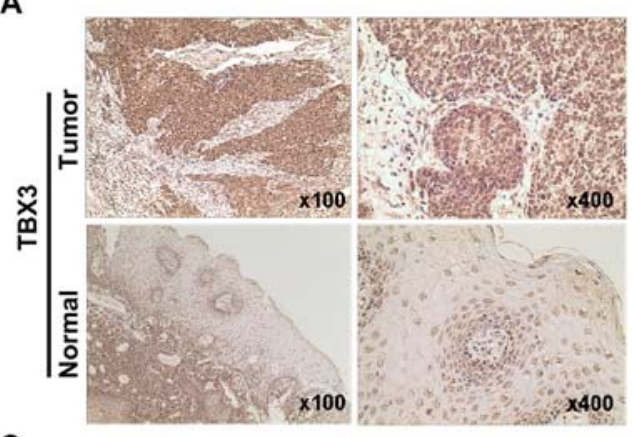

C

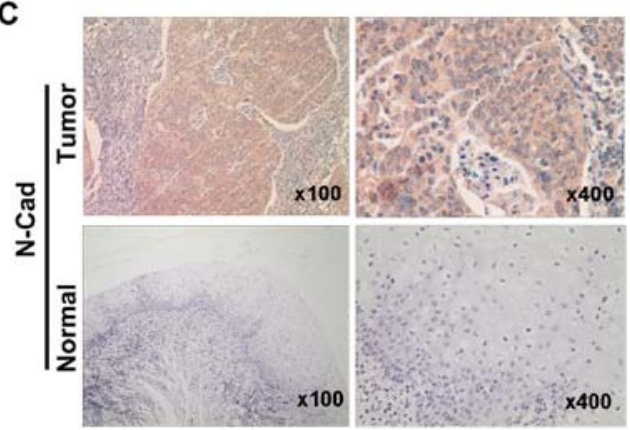

B

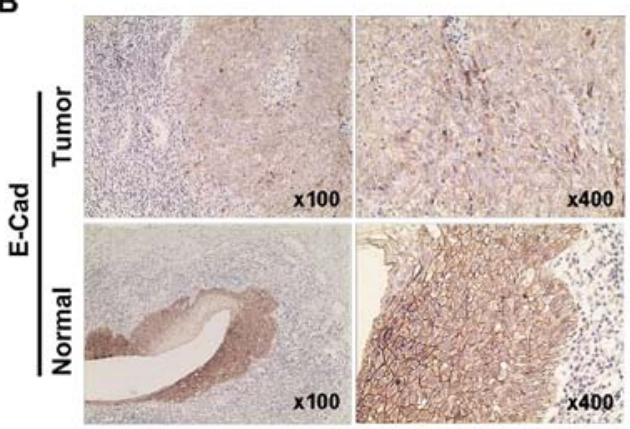

Figure 1. TBX3 and N-Cad are upregulated, whereas E-Cad is downregulated in hypopharyngeal cancer tissues. Immunohistochemical staining of (A) TBX3, (B) E-Cad and (C) N-Cad in hypopharyngeal cancer and normal tissues, all depicted by the brown staining. TBX3, T-box transcription factor TBX3; N-Cad, N-cadherin; E-Cad, E-cadherin.

A
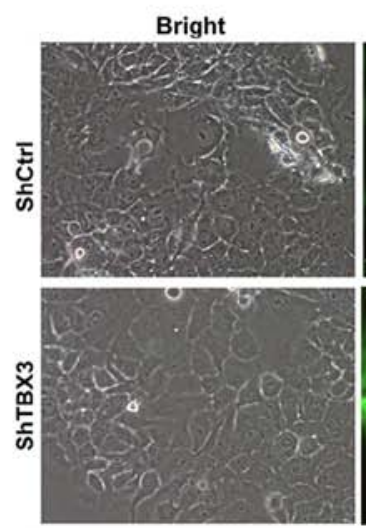

D

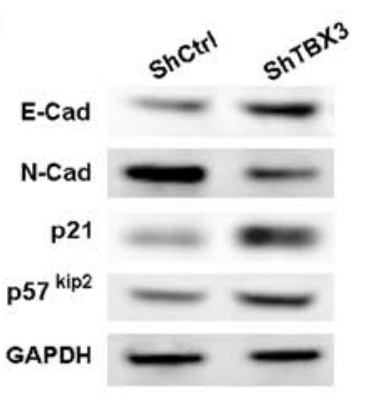

B

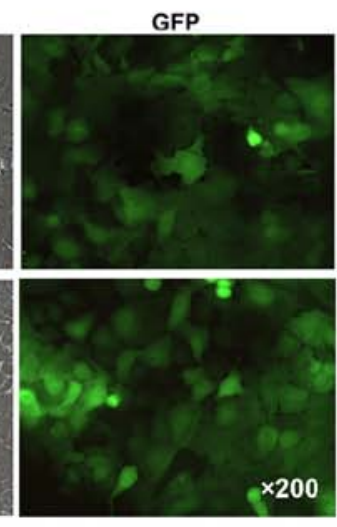

E

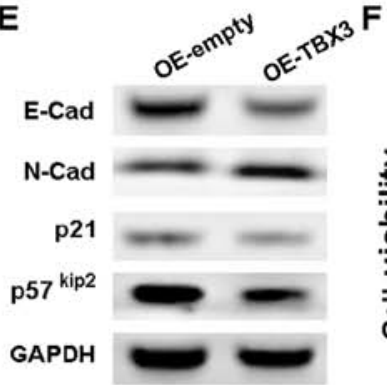

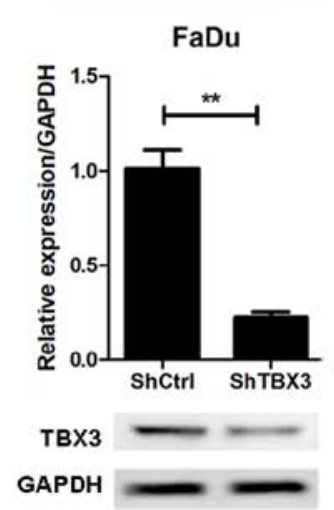

FaDu

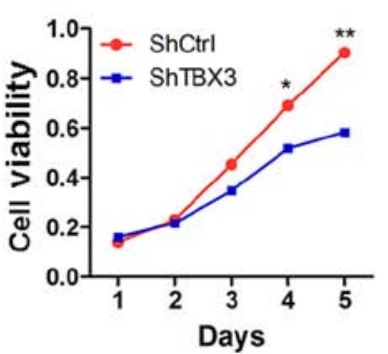

C

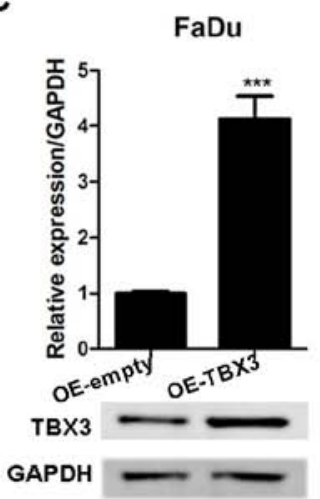

G

$\mathrm{FaDu}$

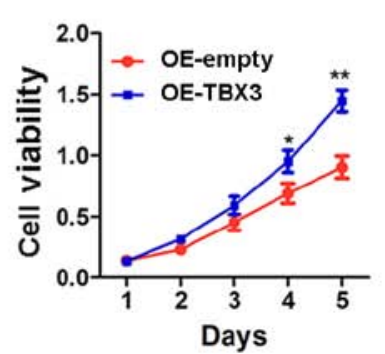

Figure 2. TBX3 expression is efficiently suppressed and overexpressed in FaDu cells using a lentivirus-mediated knockdown strategy. (A) Green fluorescence images of FaDu cells transfected with shCtrl or shTBX3 lentivirus. (B) RT-qPCR and western blotting results of TBX3 in FaDu cells expressing shCtrl or shTBX3 lentivirus ( $\left(* * \mathrm{P}<0.01\right.$ vs. shCtrl). (C) RT-qPCR and western blotting results of TBX3 in OE-empty or OE-TBX3 FaDu cells ${ }^{* * * *} \mathrm{P}<0.001$ vs. shCtrl). Western blot analysis of E-cadherin, N-cadherin, p21 and p57KIP2 in the cells described in (D) FaDu cells expressing shCtrl or shTBX3 lentivirus and (E) OE-empty or OE-TBX3 FaDu cells. An MTT assay was performed in order to detect the proliferation of the cells described in (F) FaDu cells expressing shCtrl or shTBX3 lentivirus ( $\mathrm{P}<0.05,{ }^{* * *} \mathrm{P}<0.01$ vs. shCtrl) and (G) OE-empty or OE-TBX3 FaDu cells ( ${ }^{*} \mathrm{P}<0.05,{ }^{* *} \mathrm{P}<0.01$ vs. OE-empty). TBX3, T-box transcription factor TBX3; shCtrl, short hairpin RNA control; shTBX3, short hairpin RNA targeting TBX3; RT-qPCR, reverse transcription-quantitative PCR; p21, cyclin-dependent kinase inhibitor 1; p57KIP2, cyclin-dependent kinase inhibitor 1C. 

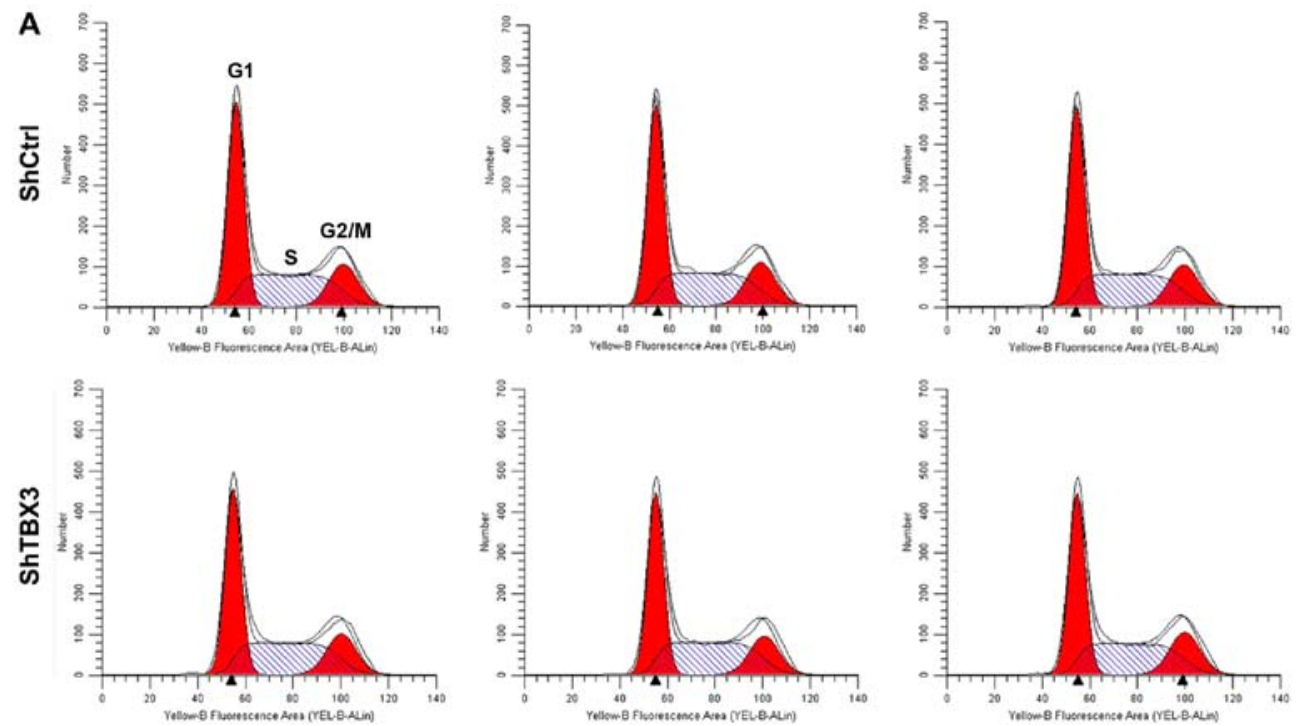

B
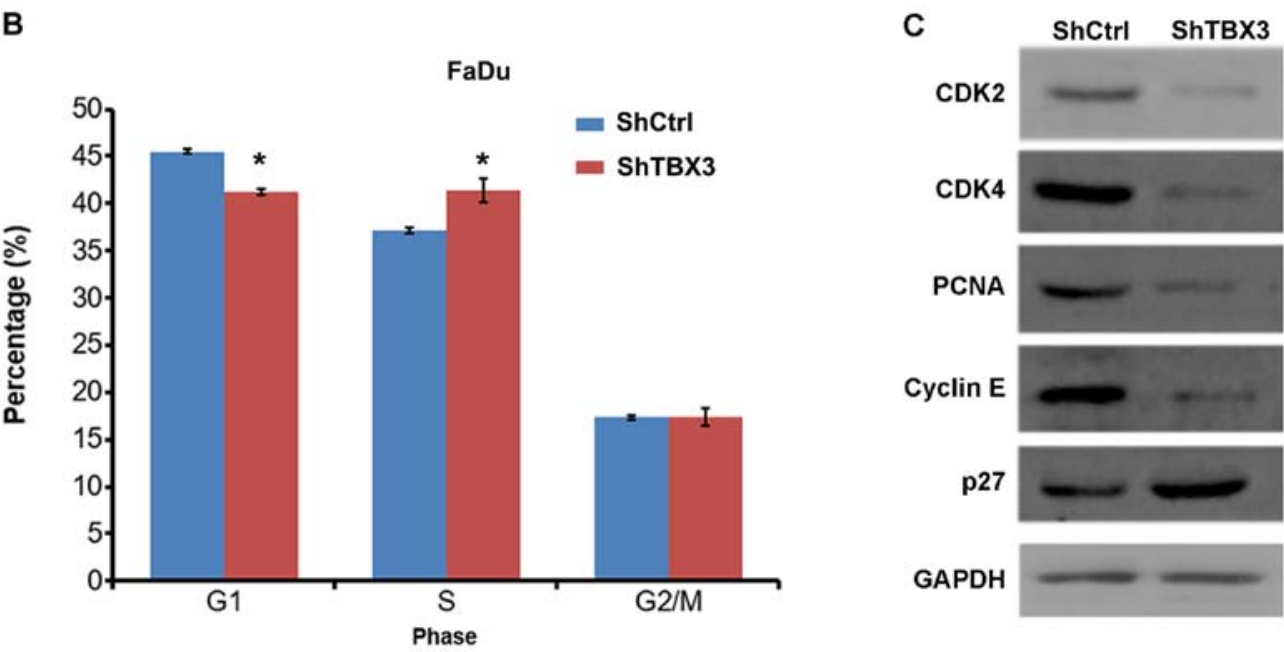

Figure 3. TBX3 silencing hinders the cell cycle progression of FaDu cells. (A and B) Flow cytometry analysis of cell cycle distribution demonstrated that TBX3 knockdown resulted in a decreased of cells in the $\mathrm{G}_{1}$ phase and an increase in the $\mathrm{S}$ phase. (C) Western blot analysis of CDK2, CDK4, PCNA, cyclin $\mathrm{E}$ and p27 in shCtrl- and shTBX3-transfected FaDu cells. "P<0.05 vs. shCtrl. TBX3, T-box transcription factor TBX3; CDK, cyclin-dependent kinase; PCNA, proliferating cell nuclear antigen; p27, cyclin-dependent kinase inhibitor 1B; shCtrl, short hairpin RNA control; shTBX3, short hairpin RNA targeting TBX3.

exact test. Spearman's correlation test was also used. A Mann-Whitney U test was applied to analyze the immunohistochemistry results. $\mathrm{P}<0.05$ was considered to indicate a statistically significant difference.

\section{Results}

TBX3 is highly expressed in cancer tissues of patients with hypopharyngeal cancer. In order to assess the clinical relevance of TBX3 in hypopharyngeal carcinoma, an immunohistochemistry assay was performed to investigate the expression of TBX3 in hypopharyngeal cancer tissue and normal hypopharyngeal specimens in the present study. The association between the expression of TBX3 and clinicopathological factors in patients with hypopharyngeal carcinoma is demonstrated in Table I. The results revealed that TBX3 protein levels were significantly increased in hypopharyngeal carcinoma compared with the normal hypopharyngeal tissues (Fig. 1A; Table II). Furthermore, the expression of N-cadherin and E-cadherin was examined in hypopharyngeal carcinoma and normal hypopharyngeal tissues. The results demonstrated a higher expression of $\mathrm{N}$-cadherin and lower expression of E-cadherin in hypopharyngeal carcinoma tissues compared with the normal hypopharyngeal tissues (Fig. 1B and C; Table II). Further analysis revealed a negative correlation between TBX3 and E-cadherin $\left(r^{2}=-0.389 ; P=0.034\right.$; Table III). Collectively, these data indicate that TBX3 is a potential biomarker for hypopharyngeal carcinoma and may be essential for the progression of this disease.

TBX3 promotes hypopharyngeal carcinoma cell proliferation. TBX3 was upregulated in hypopharyngeal carcinoma specimens, but whether it is associated with the progression of hypopharyngeal carcinoma remains less clear. In order to address this question, TBX3 was knocked-down and overexpressed in hypopharyngeal carcinoma FaDu cells, using lentivirus-based shRNA and overexpressing strategies, respectively. Green fluorescence data demonstrated that the infection efficiency was similar between the shCtrl and shTBX3 (Fig. 2A). RT-qPCR and western blotting results 

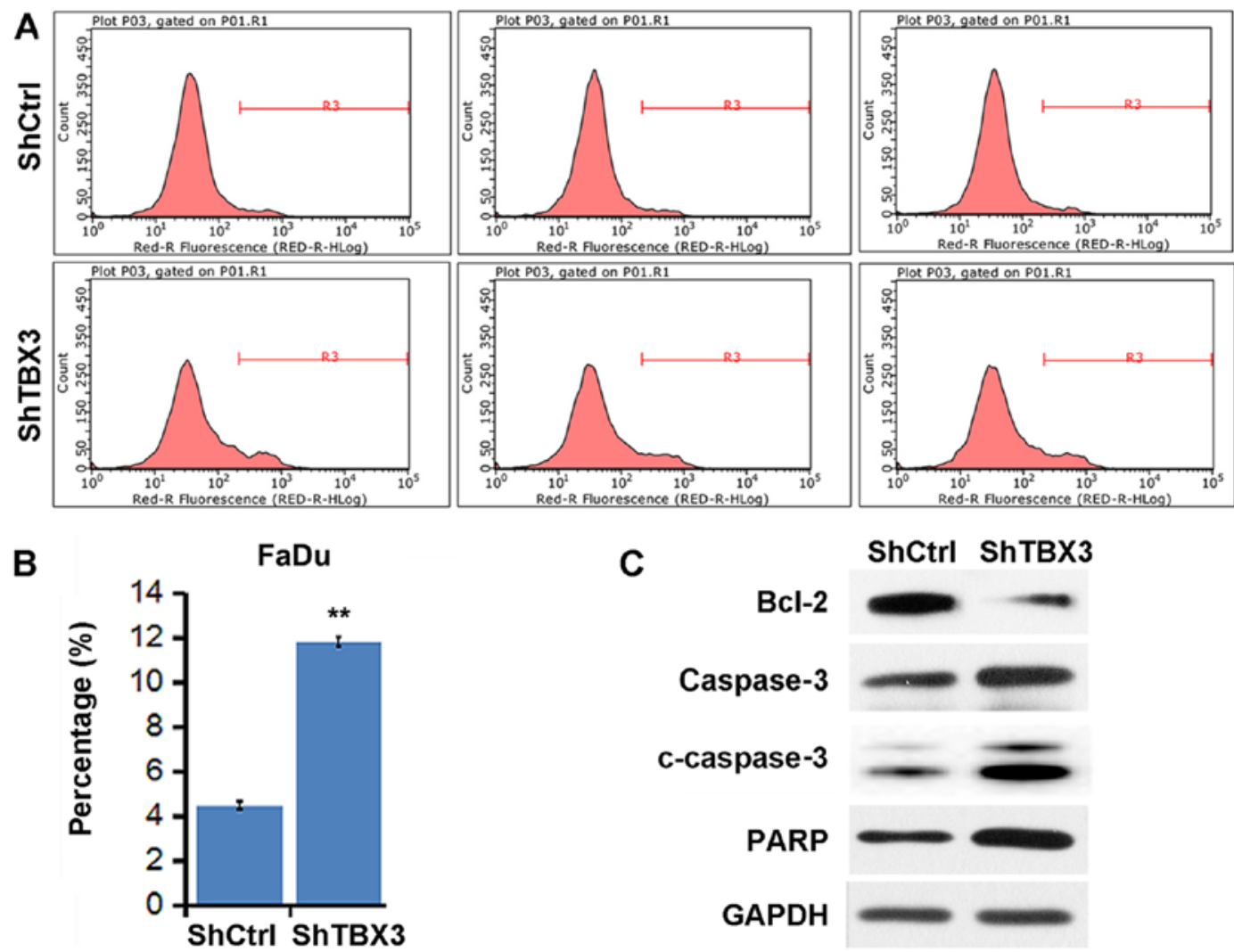

Figure 4. TBX3 silencing induces the apoptosis of FaDu cells. (A and B) Flow cytometry analysis of apoptosis revealed that TBX3 knockdown promoted FaDu cell apoptosis. (C) Western blot analysis of cleaved-caspase 3, caspase 3, Bcl-2 and PARP in shCtrl- and shTBX3-transfected FaDu cells. ** P<0.01 vs. shCtrl. TBX3, T-box transcription factor TBX3; PARP, poly (ADP-ribose) polymerase; shCtrl, short hairpin RNA control; shTBX3, short hairpin RNA targeting TBX3.

verified that TBX3 was efficiently knocked-down in the FaDu cells (Fig. 2B). In addition, TBX3 was efficiently overexpressed (Fig. 2C). The upregulation of E-cadherin, p57KIP2 and p21 was observed, as well as the downregulation of $\mathrm{N}$-cadherin in the shTBX3 group compared with shCtrl FaDu cells (Fig. 2D). The inverse results were observed in the TBX3-overexpressed cells (Fig. 2E). Subsequently, the effect of TBX3 on hypopharyngeal carcinoma cell proliferation was examined. The results of the MTT assay demonstrated that although no significant inhibition was observed on days 1 and 2, TBX3 silencing notably inhibited the proliferation of FaDu cells on days 3-5 (Fig. 2F). By contrast, TBX3 ectopic expression promoted cell proliferation (Fig. 2G). Taken together, the results from the present study demonstrate that TBX3 silencing inhibited the proliferation of hypopharyngeal carcinoma cells, indicating that this protein is critical for hypopharyngeal carcinoma cell viability.

TBX3 silencing induces cell cycle arrest in FaDu cells. Accelerated cell cycle progression is one of the main reasons that cancer cells proliferate at a higher rate than normal cells (21). The cell cycle progression in shCtrl- and shTBX3-transfected FaDu cells was investigated in the present study. Decreased $\mathrm{G}_{1}$ and increased $\mathrm{S}$ phase distribution were exhibited in shTBX3 FaDu cells compared with shCtrl cells, whereas no significant change was observed in the distribution of cells in the $\mathrm{G}_{2} / \mathrm{M}$ phase (Fig. 3A and B), indicating that the cell cycle was arrested at the $\mathrm{S}$ phase in the shTBX3-transfected FaDu cells. Furthermore, the expression of cell cycle proteins was investigated. The western blotting results revealed that CDK2, CDK4, PCNA and cyclin E expression was decreased, whereas p27 was upregulated in the shTBX3 FaDu cells (Fig. 3C). Taken together, the data from the present study demonstrated that TBX3 silencing in $\mathrm{FaDu}$ cells results in enhanced cell cycle arrest.

TBX3 silencing enhances the apoptosis rate of FaDu cells. The present study then determined whether TBX3 regulates the apoptosis rate of FaDu cells. The apoptosis rate of cells transfected with shCtrl and shTBX3 was analyzed using Annexin V-APC staining and flow cytometry. TBX3 knockdown led to an increase in the apoptosis rate of the $\mathrm{FaDu}$ cells (Fig. 4A and B). The expression of cleaved-caspase 3, caspase 3 and PARP was increased, whereas Bcl-2 was downregulated following TBX3 knockdown (Fig. 4C). These results suggest that the induction of apoptosis may contribute to suppressed cell viability in shTBX3-transfected FaDu cells.

\section{Discussion}

Although hypopharyngeal carcinoma is a relatively rare disease among the different types of head and neck cancer, it is often diagnosed when it has already reached a malignant stage (22). Treatment strategies remain ineffective against this fatal disease (22). In the last decade, certain studies have identified proto-oncogenes and tumor suppressors in hypopharyngeal carcinoma, including c-Myc (23), PI3K-mTOR (24) and phosphate and tensin homolog (PTEN) (25); however, 
the molecular mechanisms underlying the progression of hypopharyngeal carcinoma are currently poorly understood. Therefore, identifying novel targets triggering hypopharyngeal carcinoma may assist the development of effective targeted treatments.

The transcription factor TBX3, which belongs to the T-box family, is involved in the development of multiple organs, including the breast, lung and pancreas (26). Numerous studies have demonstrated that TBX3 participates in the development of different types of human cancer. For example, TBX3 serves as a downstream effector of $\mathrm{Wnt} / \beta$-catenin signaling in liver cancer growth (27). TBX3 is overexpressed in head and neck squamous cell carcinoma and represses the expression of PTEN, which is a common tumor suppressor in various types of cancer (15). Notably, TBX3 also functions as a downstream target of AKT serine/threonine kinase 3. Upregulation of TBX3 is observed in melanoma tissues and contributes to the growth and invasion of melanoma (28). At the molecular level, cell adhesion molecule E-cadherin has been reported to be inhibited by TBX3 (29,30). E-cadherin is also regulated by hepatocyte growth factor and serves as a prognostic factor for hypopharyngeal carcinoma (31). However, the association between TBX3 and E-cadherin in hypopharyngeal carcinoma remains unclear. In the present study, the involvement of TBX3 in hypopharyngeal carcinoma was investigated. First, TBX3 was observed to be significantly upregulated in hypopharyngeal carcinoma tissues, whereas E-cadherin was downregulated. In addition, $\mathrm{N}$-cadherin expression was enhanced in these tissues. This suggests that TBX3 may contribute to the progression of hypopharyngeal carcinoma. TBX3 expression was then knocked-down in FaDu cells, which are widely used to study hypopharyngeal carcinoma, using shRNA, leading to decreased cell proliferation. By contrast, TBX3 ectopic expression led to enhanced viability of the cells. Furthermore, E-cadherin and $\mathrm{N}$-cadherin were negatively and positively regulated by $\mathrm{TBX} 3$, respectively, which was consistent with the results from the patient samples in the present study. However, certain limitations remained in the present study. As it was not possible to obtain another hypopharyngeal carcinoma cell line, the functional experiments of TBX3 were not investigated in other cells. Further studies should be performed when another hypopharyngeal carcinoma cell line becomes available. Overall, the results from the present study indicate that TBX3 is a potential oncogene in hypopharyngeal carcinoma.

It has been demonstrated that TBX3 is regulated by cyclin A-CDK2 and c-Myc, and is important for S-phase progression (32). p21, a CDK inhibitor, is transcriptionally repressed by TBX3 (17). These results suggest that TBX3 is critical for the cell cycle process. In the present study, TBX3 silencing led to an increased number of cells at the $S$ phase of the cell cycle, and a decreased proportion at the $G_{1}$ phase, while the cells at the $G_{2} / M$ phase remained unchanged. Increasing evidence supports the important role that CDK2, CDK4, cyclin E and p27 serve in cell cycle progression (33-36). AsTBX3 was demonstrated to regulate the cell cycle progression of $\mathrm{FaDu}$ cells, the present study then detected whether cell cycle-associated proteins were modulated by TBX3. In the FaDu cells, CDK2, CDK4 and cyclin E were repressed following TBX3 knockdown. By contrast, p27 was upregulated by TBX3 silencing. In addition, TBX3 has been reported to regulate apoptosis via various mechanisms. This transcription factor disturbs the p53 pathway to suppress apoptosis, leading to dysregulated cell transformation and myogenic differentiation (37). TBX3 also serves as an anti-apoptotic protein in mesangial cells (38). Consistent with these results, enhanced apoptosis was observed in the TBX3-knockdown FaDu cells in the present study. Cleaved-caspase 3, PARP and Bcl-2 are markers of apoptosis. Dysregulation of these genes results in the apoptosis of cancer cells $(39,40)$. The present study then determined whether TBX3 regulated the expression of these proteins. The results revealed that cleaved-caspase 3 and PARP were upregulated, whereas the survival marker Bcl-2 was downregulated in the TBX3-silenced cells. Therefore, the results of the present study were consistent with previous studies on TBX3 in cell cycle and apoptosis regulation (41).

In conclusion, the present study provided evidence to suggest that TBX3 acts as a potential oncogene in hypopharyngeal carcinoma. Elevated TBX3 expression was observed in a cohort of hypopharyngeal carcinoma samples. Furthermore, the knockdown of TBX3 largely inhibited the proliferation of hypopharyngeal carcinoma FaDu cells and led to cell cycle arrest at the $\mathrm{S}$ phase and the induction of apoptosis. Therefore, targeting TBX3 may be a promising targeted strategy for hypopharyngeal carcinoma.

\section{Acknowledgements}

Not applicable.

\section{Funding}

No funding was received.

\section{Availability of data and materials}

The datasets used and/or analyzed during the current study are available from the corresponding author on reasonable request.

\section{Authors' contributions}

PD conceived the study, carried out the experimental design and data interpretation, and prepared and revised the manuscript. YH, HZ, XJ, YC and YZ performed the experiments. $\mathrm{RH}$ and $\mathrm{JX}$ performed the statistical analysis. All authors read and approved the final manuscript.

\section{Ethics approval and consent to participate}

Written informed consent was obtained from all participating patients, and the study was approved by the Ethics Committee of Taizhou People's Hospital (Taizhou, China).

\section{Patient consent for publication}

Not applicable.

\section{Competing interests}

The authors declare that they have no competing interests. 


\section{References}

1. Chung CH, Parker JS, Karaca G, Wu J,Funkhouser WK, Moore D, Butterfoss D, Xiang D, Zanation A, Yin X, et al: Molecular classification of head and neck squamous cell carcinomas using patterns of gene expression. Cancer Cell 5: 489-500, 2004.

2. Zvrko E, Mikic A and Vuckovic L: Clinicopathologic significance of CD105-assessed microvessel density in glottic laryngeal squamous cell carcinoma. Auris Nasus Larynx 37: 77-83, 2010.

3. Gooi Z, Richmon J, Agrawal N, Blair E, Portugal L, Vokes E, Seiwert T, de Souza J, Saloura V, Haraf D, et al: AHNS SeriesDo you know your guidelines? Principles of treatment for nasopharyngeal cancer: A review of the National Comprehensive Cancer Network guidelines. Head Neck 39: 201-205, 2017.

4. Kotwall C, Sako K, Razack MS, Rao U, Bakamjian V and Shedd DP: Metastatic patterns in squamous cell cancer of the head and neck. Am J Surg 154: 439-442, 1987.

5. Milisavljevic D, Stankovic M, Zivic M, Popovic M and Radovanović Z: Factors affecting results of treatment of Hypopharyngeal Carcinoma. Hippokratia 13: 154-160, 2009.

6. Chu PY, Wang LW and Chang SY: Surgical treatment of squamous cell carcinoma of the hypopharynx: Analysis of treatment results, failure patterns, and prognostic factors J Laryngol Otol 118: 443-449, 2004.

7. Chu PY and Chang SY: Reconstruction of the hypopharynx after surgical treatment of squamous cell carcinoma. J Chin Med Assoc 72: 351-355, 2009.

8. Kim J, Chu J, Shen X, Wang J and Orkin SH: An extended transcriptional network for pluripotency of embryonic stem cells Cell 132: 1049-1061, 2008.

9. Weidgang CE, Russell R, Tata PR, Kühl SJ, Illing A, Müller M, Lin Q, Brunner C, Boeckers TM, Bauer K, et al: TBX3 directs cell-fate decision toward mesendoderm. Stem Cell Reports 1: 248-265, 2013.

10. Russell R, Ilg M, Lin Q, Wu G, Lechel A, Bergmann W, Eiseler T, Linta L, Kumar PP, Klingenstein M, et al: A dynamic role of TBX3 in the pluripotency circuitry. Stem Cell Reports 5 : $1155-1170,2015$

11. Fan W, Huang X, Chen C, Gray J and Huang T: TBX3 and its isoform $\mathrm{TBX} 3+2 \mathrm{a}$ are functionally distinctive in inhibition of senescence and are overexpressed in a subset of breast cancer cell lines. Cancer Res 64: 5132-5139, 2004.

12. Miao ZF, Liu XY, Xu HM, Wang ZN, Zhao TT, Song YX, Xing YN, Huang JY, Zhang JY, Xu $\mathrm{H}$ and $\mathrm{Xu} \mathrm{YY}$ : Tbx3 overexpression in human gastric cancer is correlated with advanced tumor stage and nodal status and promotes cancer cell growth and invasion. Virchows Arch 469: 505-513, 2016.

13. Shan ZZ, Yan XB, Yan LL, Tian Y, Meng QC, Qiu WW, Zhang $\mathrm{Z}$ and Jin ZM: Overexpression of Tbx3 is correlated with Epithelial-Mesenchymal Transition phenotype and predicts poor prognosis of colorectal cancer. Am J Cancer Res 5: 344-353, 2014

14. Beukers W, Kandimalla R, Masius RG, Vermeij M, Kranse R, van Leenders GJ and Zwarthoff EC: Stratification based on methylation of TBX2 and TBX3 into three molecular grades predicts progression in patients with pTa-bladder cancer. Mod Pathol 28: 515-522, 2015.

15. Burgucu D, Guney K, Sahinturk D, Ozbudak IH, Ozel D, Ozbilim G and Yavuzer U: Tbx3 represses PTEN and is over-expressed in head and neck squamous cell carcinoma. BMC Cancer 12: 481, 2012.

16. Peres J, Davis E, Mowla S, Bennett DC, Li JA, Wansleben S and Prince $S$ : The highly homologous T-box transcription factors, TBX2 and TBX3, have distinct roles in the oncogenic process. Genes Cancer 1: 272-282, 2010.

17. Willmer T, Hare S, Peres J and Prince S: The T-box transcription factor TBX3 drives proliferation by direct repression of the p21(WAF1) cyclin-dependent kinase inhibitor. Cell Div 11: 6, 2016.

18. Cioffi M, Trabulo SM, Sanchez-Ripoll Y, Miranda-Lorenzo I, Lonardo E, Dorado J, Reis Vieira C, Ramirez JC, Hidalgo M, Aicher A, et al: The miR-17-92 cluster counteracts quiescence and chemoresistance in a distinct subpopulation of pancreatic cancer stem cells. Gut 64: 1936-1948, 2015.

19. Amir S, Simion C, Umeh-Garcia M, Krig S, Moss T, Carraway KL III and Sweeney C: Regulation of the T-box transcription factor Tbx3 by the tumor suppressor microRNA-206 in breast cancer. Br J Cancer 114: 1125-1134, 2016.

20. Pfaffl MW: A new mathematical model for relative quantification in real-time RT-PCR. Nucleic Acids Res 29: e45, 2001.
21. Hanahan D and Weinberg RA: Hallmarks of cancer: The next generation. Cell 144: 646-674, 2011.

22. Jang JY, Kim EH, Cho J, Jung JH, Oh D, Ahn YC, Son YI and Jeong HS: Comparison of oncological and functional outcomes between initial surgical versus non-surgical treatments for hypopharyngeal cancer. Ann Surg Oncol 23: 2054-2061, 2016.

23. Kleszcz R, Paluszczak J, Krajka-Kuźniak V and Baer-Dubowska W: The inhibition of c-MYC transcription factor modulates the expression of glycolytic and glutaminolytic enzymes in $\mathrm{FaDu}$ hypopharyngeal carcinoma cells. Adv Clin Exp Med 27: 735-742, 2018.

24. Hsu CM, Lin PM, Tsai YT, Tsai MS, Tseng CH, Lin SF and Yang MY: NVP-BEZ235, a dual PI3K-mTOR inhibitor, suppresses the growth of FaDu hypopharyngeal squamous cell carcinoma and has a synergistic effect with Cisplatin. Cell Death Discov 4: 57, 2018.

25. Liu J, Lei DP, Jin T, Zhao XN, Li G and Pan XL: Altered expression of miR-21 and PTEN in human laryngeal and hypopharyngeal squamous cell carcinomas. Asian Pac J Cancer Prev 12: 2653-2657, 2011.

26. Washkowitz AJ, Gavrilov S, Begum S and Papaioannou VE: Diverse functional networks of Tbx3 in development and disease. Wiley Interdiscip Rev Syst Biol Med 4: 273-283, 2012

27. Renard CA, Labalette C, Armengol C, Cougot D, Wei Y, Cairo S, Pineau $\mathrm{P}$, Neuveut $\mathrm{C}$, de Reyniès A, Dejean A, et al: Tbx3 is a downstream target of the Wnt/beta-catenin pathway and a critical mediator of beta-catenin survival functions in liver cancer. Cancer Res 67: 901-910, 2007.

28. Peres J, Mowla S and Prince S: The T-box transcription factor, TBX3, is a key substrate of AKT3 in melanomagenesis. Oncotarget 6: 1821-1833, 2015.

29. Feng X, Yao W, Zhang Z, Yuan F, Liang L, Zhou J, Liu S and Song J: T-box transcription factor Tbx 3 contributes to human hepatocellular carcinoma cell migration and invasion by repressing E-cadherin expression. Oncol Res 26: 959-966, 2018.

30. Du HF, Ou LP, Yang X, Song XD, Fan YR, Tan B, Luo CL and

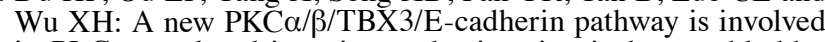
in PLCE-regulated invasion and migration in human bladder cancer cells. Cell Signal 26: 580-593, 2014.

31. Kim CH, Kim J, Kahng H and Choi EC: Change of E-cadherin by hepatocyte growth factor and effects on the prognosis of hypopharyngeal carcinoma. Ann Surg Oncol 14: 1565-1574, 2007.

32. Willmer T, Peres J, Mowla S, Abrahams A and Prince S: The T-Box factor TBX3 is important in S-phase and is regulated by c-Myc and cyclin A-CDK2. Cell Cycle 14: 3173-3183, 2015.

33. García-Reyes B, Kretz AL, Ruff JP, von Karstedt S, Hillenbrand A, Knippschild U, Henne-Bruns D and Lemke J: The emerging role of cyclin-dependent kinases (CDKs) in pancreatic ductal adenocarcinoma. Int J Mol Sci 19, 2018.

34. Xiao W, Jiang Y, Men Q, Yuan L, Huang Z, Liu T, Li W and Liu X: Tetrandrine induces G1/S cell cycle arrest through the ROS/Akt pathway in EOMA cells and inhibits angiogenesis in vivo. Int J Oncol 46: 360-368, 2015.

35. Swaffer MP, Jones AW, Flynn HR, Snijders AP and Nurse P: CDK substrate phosphorylation and ordering the cell cycle. Cell 167: 1750.e16-1761.e16, 2016.

36. Galea CA, Nourse A, Wang Y, Sivakolundu SG, Heller WT and Kriwacki RW: Role of intrinsic flexibility in signal transduction mediated by the cell cycle regulator, p27 Kip1. J Mol Biol 376: $827-838,2008$.

37. Carlson H, Ota S, Song Y, Chen Y and Hurlin PJ: Tbx3 impinges on the p53 pathway to suppress apoptosis, facilitate cell transformation and block myogenic differentiation. Oncogene 21: 3827-3835, 2002

38. Wensing LA and Campos AH: TBX3, a downstream target of TGF- $\beta 1$, inhibits mesangial cell apoptosis. Exp Cell Res 328: 340-350, 2014.

39. Warren CFA, Wong-Brown MW and Bowden NA: BCL-2 family isoforms in apoptosis and cancer. Cell Death Dis 10: 177, 2019.

40. Brown JM and Attardi LD: The role of apoptosis in cancer development and treatment response. Nat Rev Cancer 5: 231-237, 2005.

41. Li X, Ruan X, Zhang P, Yu Y, Gao M, Yuan S, Zhao Z, Yang J and Zhao L: TBX3 promotes proliferation of papillary thyroid carcinoma cells through facilitating PRC2-mediated $\mathrm{p} 57^{\mathrm{KIP} 2}$ repression. Oncogene 37: 2773-2792, 2018.

This work is licensed under a Creative Commons Attribution-NonCommercial-NoDerivatives 4.0 International (CC BY-NC-ND 4.0) License. 